\title{
The importance of physical exercise-bone mass density correlation in reducing the risk of vertebral and non-vertebral fracture in patients with osteoporosis
}

\author{
Sinziana Calina Silisteanu ${ }^{1}$, Andrei Emanuel Silisteanu ${ }^{2}$ \\ ${ }^{1}$ Railway Hospital Iasi - Specialty Ambulatory of Suceava - "Stefan cel Mare" University of Suceava
} FEFS-DSDU

Coresponding author: Sinziana Silisteanu

E-mail address: sinzi_silisteanu@yahoo.com

\begin{abstract}
Introduction. Osteoporosis is a systemic health condition, predominantly in the skeleton, characterized by the decreasing bone strength and bone density as well as by the modification of this structure, being prone to an increased fracture risk. The bone strength of the bone depends on the density and quality of the bone. The bone density was measured by DEXA imagery investigation which uses the dual absorptiometry by X-rays.Material and method. The study was conducted over a period of one year on a total of 30 patients diagnosed with osteoporosis, age 50-70 years. For the assessment of the pain, the visual analogue-VAS scale was used to assess the quality of life - the Qualeffo-41 questionnaire and for assessing fracture risk - the FRAX scorer. The bone mineral density was assessed by the DEXA assay in the lumbar spine and femur using the T score. The patients received pharmacological treatment with bisphosphonates and performed a kinetotherapy program 2 times a week.Results. Patients participating in the study were 60 years old. The repartition depending on the life environment has revealed a greater frequency of the disorder in rural areas than the urban area. The values of the T-student test were statistically significant for the spine $(p=0.0006)$ and for the hip $(p=0.03)$ Conclusions. Research was based on the use of diagnostic utility indices such as bone mineral density, data that correlate with DEXA investigation and fracture risk. Determination of fracture risk with the FRAX score is important in order to identify those patients who need therapy and at the same time to assess the efficiency/cost ratio for the applied therapy. Osteoporosis is a public health problem requiring early diagnosis to optimize therapeutic strategies
\end{abstract}

Key words: osteoporosis, bone mineral density, biphosphonates, physical activity

\section{Introduction}

Osteoporosis is a systemic health condition, predominantly in the skeleton, characterized by the decreasing bone strength and bone density as well as by the modification of this structure, being prone to an increased fracture risk. The bone strength of the bone depends on the density and quality of the bone. The bone density was measured by DEXA imagery investigation which uses the dual absorptiometry by $\mathrm{X}$-rays. The DEXA exam is a fast test (approximately 10 minutes) that is made at the level of the lumbar spine at the L1-L4 vertebras and at the level of the coxofemural joint by analysing the bone mass density (BMD). After the mass bone analysis by means of DEXA, osteoporosis is defined by values with -2.5 deviations in comparison to the normal value of the adult for the age of the chosen bone density. The fracture risk can also be calculated by the FRAX test. The clinical factors for the occurrence of fractures are: the patient's age over 40 , the female gender, the small waist, previous fractures, smoking, alcohol consumption, medication intake, especially glucocorticoids[1]. Another investigation is the calculation of a score, namely the trabecular bone score. It is made by the TBS method that is a 3D quantity analysis of the lumbar spine by the bidimensional projection of a DEXA investigation, requiring a special soft installed on the DEXA devices [2]. TBS offers information about the bone micro-architecture. There are studies [3] which show that the TBS predicts more accurately the fracture bone in comparison to the measurements of the BMD at the level of the lumbar spine. Also, the TBS is associated to a higher fracture risk for the persons with reduced bone mass, being influenced only by the poor quality of the bone. There were situations when the values were normal by the simple DEXA whereas TBS indicated osteoporosis. As for the osteoporosis that occurs to the patients with diabetes mellitus, the bone fracture assessed by DEXA was three times lower in comparison to the TBS test. Another finding was the increase of the BMD assessed by DEXA by the use of bisphosphonates but no increase of TBS. Therefore, it is considered that the most efficient treatments are not the ones that determine the increase of the BMD but the ones that increase the TBS. [2] Other research [4] proved that it is recommended to the elder persons to do physical exercises provided 
that the programme is not too a very tough one since it makes vertebral modifications: the bone density measured by DEXA increases but the TBS remains unchanged. This is why it is proposed to consider the use of the trabecular bone score (TBS) and not only the use of the bone mass density (BMD).

The results of a trial published in 2017 [5] indicates that in Taiwan, in the rural areas, the prevalence of the osteopenia and of osteoporosis for the women who already had menopause and for the men over 65 is rather high whereas these persons get medication but their bone health does not improve considerably. The trial had in view 941 adults (651 women and 290 men) out of which $63.7 \%$ had modified bone density, namely $46.9 \%$ had osteopenia and 16,8\% had osteoporosis.

In 2012 AHRQ [6] presented the benefits and the risks of the medication drunk on order to prevent and treat osteoporosis: bisphosphonates (alendronate, risedronate, ibandronate, zolendronate acid), calcitonin, estrogens, selective modulators of the estrogen receptors (Raloxifene) and denosumab (approved in 2010 by FDA). The purpose was to prove the role of the calcium and of the $\mathrm{D}$ vitamin in food and as supplements; moreover, the physical exercises are useful for preserving the bone mass, thus decreasing the fracture risk for the patients diagnosed of osteoporosis. The treatment by $\mathrm{D}$ vitamin decreased the risk of vertebral fracture for the patients who have primary osteoporosis. The sole intake of calcium does not decrease the risk of vertebral fracture in comparison to the must treatment of calcium and D vitamin.

Another trial [7] indicated that the intake of alendronate, risendronate, zolendronate acid and denosumab prevents the occurrence of hip fracture for the women who were diagnosed of osteoporosis and it decreases the risk of vertebral fractures. In addition, the intake of risendronate decreases the risk of vertebral and non-vertebral fractures for the men diagnosed of osteoporosis. The results of a metaanalysis [8] published in 2015 indicated that the use of bisphosphonates and denosumab for the patients diagnosed of postmenopausal osteoporosis decreases the risk of fractures. Risendronate, alendronate and denosumab are very efficient in the decrease of the vertebral and non-vertebral fracture whereas the risendronate can decrease the risk of arm fractures.

In 2013 a published article emphasized the result of 34 trials by which it was found out that the denosumab, risendronate and acid zolendronate reduced significantly the risk of vertebral and nonvertebral fractures whereas the alendronate and the strontium ranelate reduced the risk of vertebral fractures. Moreover, these comparative trials proved that the denosumab is more efficient in preventing the new vertebral fractures in comparison to the strontium ranelate, alendronate and risendronate [9 ,10,11].

A trial published in 2017 outlined the importance of the individualized physical activity in improving the femoral bone health [12,13] preventing fractures and improving the quality of life of patients with osteoporosis.

The results of 13 studies have shown that a moderatehigh physical activity is associated with a reduction in the risk of hip fracture by $45 \%$ and with the decrease of the risk of falling into active people. [14] It has been found that exercise and recovery programs are effective in preventing falls or bone loss. Over $90 \%$ of hip fractions are due to falling $[15,16]$.

A study investigated the association of daily household activity, physical activity in leisure time, professional activity and the risk of hip fracture. During 13 years, over 23,000 persons over 50 of both sexes were investigated. It was found that people who had physical activity under one hour / week were at 85\% risk of hip fracture [17].

Another study involving over 14,000 people over 60 has shown that reducing physical activity is associated with an increased risk of hip fracture [18] .Obesity has been associated with high bone density and low fracture risk. However, the body mass index was correlated with the risk of hip fracture, especially in women. Maintaining the bone health is essential in reducing the risk of osteoporosis and the occurrence of potential disabilities. Among the factors involved in the bone structure modification are food factors, food intake, and magnesium, calcium, potassium supplements to help prevent osteoporosis [19] .The diet rich in magnesium may have a role in preventing, alongside with the aging, the fall of the muscle mass, of the muscle strength [20]. The alkaline diet correlates with the age, the physical activity, and protein intake to help prevent the muscle loss [21]. Besides calcium and vitamin $\mathrm{D}$, there are other micronutrients (iron, copper, selenium) that help prevent osteoporosis. By increasing the amounts of fruits and vegetables, which contain micronutrients and are useful for bone remodeling, to 5 portions a 
day, can influence the bone health. The balanced diet and the physical activity can help prevent osteoporosis [22,23]. The calcium intake has a positive effect on bone mass, may reduce the risk of falling and of fracture [24,25, 26].

\section{OBJECTIVES}

The study sought to highlight the link between the physical activity and the bone mass in order to reduce the risk of fracture in patients with osteoporosis.

\section{MATERIAL AND METHOD}

The study was conducted over a period of one year on a total of 30 patients diagnosed with osteoporosis. The research was carried out in an outpatient regime, complying with the ethical and deontological principles. The inclusion criteria in the study were: patients with a confirmed diagnosis of osteoporosis and who accepted the study, age $50-70$ years, with no decompensated chronic disease. Exclusion criteria: Non-cooperative patients who did not give the consent to participate in the study, under 50 and over 70 , people with decompensated chronic conditions. The assessment of the patients we included demographic variables such as age, sex, life environment, body mass index. For the assessment of the pain, the visual analogue-VAS scale was used to assess the quality of life - the Qualeffo-41 questionnaire and for assessing fracture risk - the FRAX scorer.

The bone mineral density was assessed by the DEXA assay in the lumbar spine and femur using the $\mathrm{T}$ score. The patients received pharmacological treatment with bisphosphonates and performed a kinetotherapy program (active-passive mobilizations, mediumintensity and medium-strength actives) 2 times a week. For the statistical analysis the t-student test was used to compare the groups, the value for statistical significance $\mathrm{p}$ being less than 0.05 .

\section{RESULTS}

Patients participating in the study were 60 years old (60 \pm 6.1251 ). The repartition depending on the life environment has revealed a greater frequency of the disorder in rural areas with 18 patients (60\%) than the urban area with 12 patients (40\%). The average weight of the patients was $73 \mathrm{~kg}$, the average height was $158 \mathrm{~cm}$, the Body Mass Index had an initial average value of 29.25 and an average final value of 28.98.
The bone mineral density at baseline in the lumbar spine and the femoral area expressed by the $\mathrm{T}$ test allowed the following data to be obtained:

- for the lumbar spine, $\mathrm{T}$ had for 16 patients (53.34\%) values in the range $-2.5 \div-3$, for 8 patients(26.67\%) values in the range $-3 \div-3.5$, for 2 patients $(6.67 \%)$ values between $-3.5 \div-4$ and for 4 patients $(13.34 \%)$ values over -4

- for the hip, $\mathrm{T}$ had for 24 persons (80\%) values below -2.5 , and for 2 persons (6.67\%) values in the ranges $2.5 \div 3,-3 \div 3.5,-3.5 \div 4$

The bone mineral density determined at the final moment had the following data:

- for the lumbar spine, T had for 6 patients (20\%) values below -2.5 , for 10 patients (33.34\%) values in the range $-2.5 \div-3$, for 8 patients $(26.67 \%)$ values in the range $-3 \div-3.5$, for 2 patients $(6.67 \%)$ values between-3.5 $\div-4$ and for 4 patients $(13.34 \%)$ values over -4

-for the hip, $\mathrm{T}$ had for 26 persons (86.67\%) values below -2.5 , and for 2 persons (6.67\%) values in the ranges $-2.5 \div 3$ and -4 .

The average T-score for the lumbar spine was initially $-2.9 \pm 0.71$ and finally $-2.9 \pm 0.69$.

The average T-score for the hip was initially $-1.5 \pm$ 1.08 and finally $-1.1 \pm 1.03$.

For the assessment of the fracture risk, we used the FRAX score that revealed a major osteoporotic fracture risk in the initial 10 years of initially $3.7 \pm$ 3.24 and final of $3.7 \pm 4.06$ and a risk of an initial fracture of $0.7 \pm 2.15$ and final of $0.5 \pm 2.94$.

The values of the T-student test were statistically significant for the spine $(p=0.0006)$ and for the hip $(\mathrm{p}=0.03)$

\section{CONCLUSIONS}

- Research was based on the use of diagnostic utility indices such as bone mineral density, data that correlate with DEXA investigation and fracture risk

- Determination of fracture risk with the FRAX score is important in order to identify those patients who need therapy and at the same time to assess the efficiency/cost ratio for the applied therapy

- The FRAX score can give reliable data for the risk of a fracture occurring within the next 10 years at the vertebral and non-vertebral level

- Osteoporosis is a public health problem requiring early diagnosis to optimize therapeutic strategies 


\section{REFERENCES}

1. Peretianu D, Poiana C , Controverse legate de DEXA,Viata medicala,7 (1361)12 ian 2016

2. Silva BC et al. Trabecular bone score: a noninvasive analytical method based upon the DXA image. J Bone Miner Res. 2014 Mar;29(3):518-30

3. Silva BC et al. Trabecular bone score (TBS)--a novel method to evaluate bone microarchitectural texture in patients with primary hyperparathyroidism. J Clin Endocrinol Metab. 2013 May;98(5):1963-70

4. Heiniö L et al. Association between long-term exercise loading and lumbar spine trabecular bone score (TBS) in different exercise loading groups. J Musculoskelet Neuronal Interact. 2015 Sep;15(3):279-85

5. Chen PH, Lin MS, Huang TJ, Chen MY., Prevalence of and factors associated with adopting bone health promoting behaviours among people with osteoporosis in Taiwan: a cross-sectional study, BMJ Open. 2017 Sep 25;7(9):e015980. doi: 10.1136/bmjopen-2017015980.

6. Levis S, Theodore G, Summary of AHRQ's comparative effectiveness review of treatment to prevent fractures in men and women with low bone density or osteoporosis: update of the 2007 report, J Manag Care Pharm. 2012 May;18(4 Suppl B):S1-15; discussion S13.

7. Crandall CJ, Newberry SJ, Diamant A, Lim YW, Gellad WF, Suttorp MJ, Motala A, Ewing B, Roth $B^{1}$, Shanman R, Timmer M, Shekelle PG, Treatment To Prevent Fractures in Men and Women With Low Bone Density or Osteoporosis: Update of a 2007 Report [Internet], Rockville (MD): Agency for Healthcare Research and Quality (US); 2012 Mar. Report No.: 12-EHC023-EF. AHRQ Comparative Effectiveness Reviews.

8. Zhang L, Pang Y, Shi Y, Xu M, Xu X, Zhang $\mathrm{J}$, Ji L, Zhao D, Indirect comparison of teriparatide, denosumab, and oral bisphosphonates for the prevention of vertebral and nonvertebral fractures in postmenopausal women with osteoporosis, Menopause. 2015 Sep;22(9):1021-5. 10.1097/GME.0000000000000466
9. Freemantle N, Cooper C, Diez-Perez A, Gitlin M, Radcliffe H, Shepherd S, Roux C, Results of indirect and mixed treatment comparison of fracture efficacy for osteoporosistreatments: a meta-analysis, Osteoporos Int. 2013 Jan;24(1):209-17. doi: 10.1007/s00198-0122068-9. Epub 2012 Jul 26.

10. Hopkins RB, Goeree R, Pullenayegum E, Adachi JD, Papaioannou A, Xie F, Thabane L, The relative efficacy of nine osteoporosis medications for reducing the rate of fractures in post-menopausal women, BMC Musculoskelet Disord. 2011 Sep 26;12:209. doi: 10.1186/1471-2474-12-209.

11. Bilezikian JP, Efficacy of bisphosphonates in reducing fracture risk in postmenopausal osteoporosis, Am J Med. 2009 Feb;122(2 Suppl):S14-21. doi: 10.1016/j.amjmed.2008.12.003.

12. Fuchs RK, Kersh ME, Carballido-Gamio J, Thompson $\mathrm{WR}^{1}$, Keyak JH, Warden SJ, Physical Activity for Strengthening Fracture Prone Regions of the Proximal Femur, Curr Osteoporos Rep. 2017 Feb;15(1):43-52. doi: 10.1007/s11914-017-0343-6.

13. Miyakoshi N, Daily practice using the guidelines for prevention and treatment of osteoporosis. Effectiveness of exercise for preventing and treating osteoporosis, Clin Calcium. 2008 Aug;18(8):1162-8. doi: CliCa080811621168.

14. Moayyeri A, The association between physical activity and osteoporotic fractures: a review of the evidence and implications for future research, Ann Epidemiol. 2008 Nov;18(11):827-35. doi: 10.1016/j.annepidem.2008.08.007. Epub 2008 Sep 21.

15. Abe S, Narra N, Nikander R, Hyttinen J, Kouhia $\mathrm{R}$, Sievänen $\mathrm{H}$, Exercise loading history and femoral neck strength in a sideways fall: A threedimensional finite element modeling study, Bone. $2016 \quad$ Nov;92:9-17. doi: 10.1016/j.bone.2016.07.021. Epub 2016 Jul 28

16. Nikander $R$, Kannus $P$, Dastidar $P$, Hannula M, Harrison L, Cervinka T, Narra NG, Aktour $\mathrm{R}$, Arola T, Eskola H, Soimakallio S, Heinonen A, Hyttinen J, Sievänen $\mathrm{H}$, Targeted exercises against hip fragility, Osteoporos Int. 2009 Aug;20(8):1321-8. doi: 10.1007/s00198-0080785-x. Epub 2008 Nov 11 
17. Lagerros YT, Hantikainen E, Michaëlsson K, Ye W, Adami HO, Bellocco R., Physical activity and the risk of hip fracture in the elderly: a prospective cohort study, Eur J Epidemiol. 2017 Nov;32(11):983-991. doi: 10.1007/s10654-0170312-5. Epub 2017 Sep 22.

18. Moayyeri A, Besson H, Luben RN, Wareham NJ, Khaw KT, The association between physical activity in different domains of life and risk of osteoporotic fractures, Bone. 2010 Sep;47(3):693-700. doi: 10.1016/j.bone.2010.06.023. Epub 2010 Jun 28.

19. Hayhoe RP, Lentjes MA, Luben RN, Khaw KT, Welch AA, Dietary magnesium and potassium intakes and circulating magnesium are associated with heel bone ultrasound attenuation and osteoporotic fracture risk in the EPICNorfolk cohort study, Am J Clin Nutr. 2015 Aug;102(2):376-84.

doi: 10.3945/ajcn.114.102723. Epub 2015 Jul 1.

20. Welch AA, Kelaiditi E, Jennings A, Steves CJ, Spector TD, MacGregor A., Dietary Magnesium Is Positively Associated With Skeletal Muscle Power and Indices of Muscle Mass and May Attenuate the Association Between Circulating C-Reactive Protein and Muscle Mass in Women, $\mathrm{J}$ Bone Miner Res. 2016 Feb;31(2):317-25. doi: 10.1002/jbmr.2692. Epub 2015 Sep 11.

21. Welch AA, MacGregor AJ, Skinner J, Spector TD, Moayyeri A, Cassidy A, A higher alkaline dietary load is associated with greater indexes of skeletal muscle mass in women, Osteoporos Int. 2013 Jun;24(6):1899-908. doi: 10.1007/s00198-012-2203-7. Epub 2012 Nov 14.

22. Antonescu E, Totan M, Boitor GC, Szakacs J, Silisteanu SC, Fleaca SR, Cernusca Mitariu B, Serb $H$, The Reference Intervals Used in Pediatric Medical Analysis Laboratories to Interpret the Results Analysis for Total Serum Calcium, Revista de chimie 68 (2), 243245,http://www.revistadechimie.ro/pdf/ANTON ESCU

23. Higgs J, Derbyshire E, Styles K, Nutrition and osteoporosis prevention for the orthopaedic surgeon: A wholefoods approach, EFORT Open Rev. 2017 Jun 23;2(6):300-308. doi: 10.1302/2058-5241.2.160079. eCollection 2017 Jun.
24. Antonescu E, Szakács J, Totan M, Determination of reference intervals for total calcium and magnesium specific for children and adolescents in Sibiu area, Revista Romana de Medicina de Laborator $24 \quad$ (3), 347-350, DOI: https://doi.org/10.1515/rrlm-2016-0030

25. Osteoporosis: the role of micronutrients, Am J Clin Nutr. 2005 May;81(5):1232S-1239S.

26. Lanham-New SA, Importance of calcium, vitamin $\mathrm{D}$ and vitamin $\mathrm{K}$ for osteoporosis prevention and treatment, Proc Nutr Soc. 2008 May;67(2):163-76. doi:Nieves JW 10.1017/S0029665108007003. 